\section{How Are We Doing in Reviewing Those New Patients Who Need to Be Seen as Early as Possible? An Audit of 264 Consecutive New Patients Seen Over 6 Months in a University Hospital in Ireland}

\section{To the Editor:}

We read with interest the article entitled "Triage of referrals to an outpatient rheumatology clinic: analysis of referral information and triage" ${ }^{\text {, and }}$ agree that there are continuing difficulties to ensure effective stratification of rheumatology referrals. We would like to share our own experience in dealing with this uphill task.

In our 4 weekly clinics, new patient slots are kept reserved for new patients who potentially have early inflammatory joint disease (IJD). To allocate early appointments for such new patients, the 2 consultants here use a triage system in an attempt to see patients with early IJD within 8 weeks of receipt of the general practitioner referral letter. They carefully review these letters and allocate new appointments based on the information contained therein. Correspondence is then sent to the general practitioners giving them the date of the appointment and informing them that we triage appointments based on the information they give us in their referral letters.

However, we understand that performance measurement is a fundamental building block to an effective patient care model. Keeping this in mind, we conducted a retrospective review of all 264 new patients seen over 6 months in our clinics (January 1-June 30, 2007). Our objectives were 2-fold. First, we wanted to examine the effectiveness of our triage system in seeing early, within 8 weeks of receipt of the GP referral letter, new patients with IJD. And second, we wished to investigate the underlying reasons for any delays in patients with IJD being given "new/first-visit" patient appointments beyond our target timeframe. By chart review in 2008, we identified those patients with definite IJD. Demographic data, date of GP referral letter, date of first review, and the final rheumatologic diagnosis were documented. Sixty-three patients with IJD were identified (i.e., $24 \%$ of new outpatient referrals had IJD).

Our results showed that 44 patients $(70 \%)$ were seen within 8 weeks of receipt of the GP referral letter, 27 (43\%) were seen within 4 weeks, and 17 (27\%) within 2 weeks. Eleven patients were seen 3-6 months after referral; we reviewed the referral letters of this subgroup and found that they were missing crucial clinical information (especially morning stiffness, duration of symptoms, swollen and tender joints) and laboratory data that would have helped the authors triage more appropriately.

This audit shows that our system is reasonably effective in prioritizing and allocating appointments for new patients with early IJD. We can do better. This audit also suggests that education of general practitioners about what to include in their referral letters would help us better triage new patient appointments; if the GP had given more pertinent details, it would have resulted in even more patients with early IJD being seen earlier then they were.

MUHAMMAD HAROON, MBBS, MRCPI, MMedSc, Rheumatology Specialist, Registrar; URSULA BOND, RNP, Hip Dip Rheumatology; MARK PHELAN, MB, BCh, BAO, MRCP (UK); MICHAEL REGAN, FRCP (UK), FRCPI, FACP, FACR, Department of Rheumatology, South InfirmaryVictoria University Hospital, Cork, Ireland. Address reprint requests to Dr. Haroon. E-mail: mharoon301@ @otmail.com

\section{REFERENCE}

1. Graydon SL, Thompson AE. Triage of referrals to an outpatient rheumatology clinic: analysis of referral information and triage. J Rheumatol 2008;3:1378-83.

J Rheumatol 2009;36:2; doi:10.3899/jrheum.080789 